\title{
Nanotecnología en la periferia: los casos de Costa Rica, Uruguay y Venezuela
}

\section{Nanotechnology on the periphery: the cases of Costa Rica, Uruguay and Venezuela}

\author{
Adriana Chiancone, ${ }^{* *}$ María Sonsiré López, ${ }^{* *}$ José Roberto Vega Baudrit***
}

\begin{abstract}
This paper addresses nanotechnology developments in three Latin American countries: Costa Rica, Uruguay and Venezuela. It aims both to characterize the field in these countries and to develop a first comparative approach. The methodology adopted included documentary analysis and analysis of specialized databases, mainly the StatNano database. The article describes and compares the incorporation of nanotechnology in the public agenda and policies; policy instruments, financing, governance and nanosecurity. Moreover, research and development (R\&D) activities are characterized in terms of scientific production and technological innovation. The results of the study showed that the last two decades have been the time frame of a process of increasing institutionalization and internationalization of nanotechnology in this group of countries, where this field has primarily followed national patterns but faces common challenges.
\end{abstract}

KEYWORDS: nanotechnology, international comparison, Costa Rica, Uruguay, Venezuela.

RESUMEN: Este trabajo aborda la situación actual de la nanotecnología en tres países latinoamericanos: Costa Rica, Uruguay y Venezuela. Se busca caracterizar el trabajo en este campo en esos países y desarrollar una primera aproximación de carácter comparativo. La metodología adoptada incluyó revisión documental y análisis de bases de datos especializadas, fundamentalmente la base StatNano. El artículo describe y compara en los tres países la incorporación de la nanotecnología en la agenda y en las políticas públicas; instrumentos de política, financiamiento, gobernanza y nanoseguridad. Se caracterizaron, asimismo, las actividades de investigación y desarrollo $(1+D)$ en términos de producción científica y de innovación tecnológica. Del estudio realizado surge que las últimas dos décadas han sido el marco temporal de un proceso de creciente institucionalización e internacionalización en el área de la nanotecnología en los países considerados, en los cuales el desarrollo de este campo ha seguido fundamentalmente pautas nacionales, pero enfrenta desafíos comunes.

PALABRAS CLAVE: nanotecnología, comparación internacional, Costa Rica, Uruguay, Venezuela.

\section{Introducción}

En este artículo se aborda la situación de las nanociencias y las nanotecnologías (a las que nos referiremos a lo largo de este trabajo como nanotecnología)

Recibido: 7 de junio 2021.

Aceptado: 31 de julio 2021.

Publicado: 25 de agosto 2021.

\footnotetext{
* Universidad de la República, Uruguay.

** Instituto Venezolano de Investigaciones Científicas, Venezuela.

*** LANOTEC, Centro Nacional de Alta Tecnología, Costa Rica.

-Autora de correspondencia: achiancouniversidad@gmail.com
} 
en tres países latinoamericanos: Costa Rica, Uruguay y Venezuela. En el título del artículo, hemos empleado la expresión 'nanotecnología en la periferia', aludiendo a los cambios en las interpretaciones de la dinámica centro-periferia.

El concepto "ciencia periférica" ha sido ampliamente utilizado para dar cuenta de los procesos de producción y apropiación de conocimiento, y de las relaciones entre "centro" y "periferia". En el contexto de revisiones y problematizaciones de esta noción, se ha destacado su carácter ahistórico y estático, así como también las limitaciones de su capacidad explicativa. Esto ha llevado a pasar de enfoques que consideran la pertenencia al "centro" o a la "periferia" como factores explicativos, a otros donde estos conceptos son interpretados como consecuencia de procesos relacionales creados y reproducidos tanto social como cognitivamente (Matharan, 2017: 37-40).

Un concepto alternativo como el de "ciencia en la periferia", planteado por Marcos Cueto (1989), implica que "el trabajo científico en estos países tiene sus propias reglas, que deben ser entendidas no como síntomas de atraso o modernidad, sino como parte de su propia cultura y de las interacciones con la ciencia internacional" (Cueto, 1989: 29). A diferencia del concepto "ciencia periférica”, el concepto de ciencia en la periferia permite visualizar la heterogeneidad al interior de las particulares comunidades científicas, así como entre varias comunidades científicas ubicadas en contextos con notorias diferencias (Kreimer, 2010: 44)

Con la creciente interconexión global aumenta la diferenciación en las relaciones centro-periferia (Hanners, 2015). Como en otros campos del conocimiento, existe heterogeneidad en los desarrollos de la nanotecnología en América Latina, y pueden observarse importantes diferencias entre los países y al interior de estos.

La investigación en nanotecnología y la focalización en temas distintos han sido configuradas por la diferentes formas de inserción en las redes internacionales, regionales o nacionales. A su vez, el grado de desarrollo de la estructura de investigación y recursos humanos condiciona las formas de inserción en esas redes. En la medida en que la distribución en la región de esas estructuras y recursos es desigual, los países de mayor tamaño están generando mayores capacidades autónomas (Invernizzi, Hubert y Vinck, 2014: 19).

Brasil, México y Argentina, que se destacan ampliamente en nanotecnología en términos de capacidades instaladas, recursos humanos, financiamiento, así como de visibilidad de sus actividades de investigación y desarrollo (I+D), son precisamente los países latinoamericanos más estudiados, más allá de la producción regular de trabajos sobre diferentes aspectos de la nanotecnología en la región (Foladori e Invernizzi, 2008 y 2013; Foladori et al., 2012 y 2016; RICYT, 2008; Kay y Shapiro, 2009; entre otros).

En este contexto conceptual, cabe preguntarse cuál es la situación en países con menores posibilidades en la nanotecnología, un campo que requiere de equipamiento e infraestructura de alto costo, así como de recursos humanos altamente capacitados, y donde, consecuentemente, existe una 
gran dependencia del financiamiento y de las posibilidades de acceso a instrumentos y personal idóneos.

En este artículo se busca caracterizar el trabajo en nanotecnología en Costa Rica, Uruguay y Venezuela y desarrollar una primera aproximación de carácter comparativo.

La comparación de sus respectivos desarrollos en este campo y de algunos productos científicos y de innovación, permite identificar similitudes y diferencias entre sus dinámicas de creación y cambio, así como identificar factores que se asocian con dicha heterogeneidad.

El trabajo se estructura en cuatro secciones. La primera presenta los aspectos metodológicos del estudio. En la segunda se ubica la situación de la nanotecnología en cada país, en el marco del desarrollo más general de la ciencia, tecnología e innovación (CTI). Las características de los países en cada uno de los ejes elegidos para la comparación son expuestas en la tercera sección. El artículo se cierra con una sección de discusión.

\section{Aspectos metodológicos}

La perspectiva comparativa recae sobre tres países latinoamericanos que no pertenecen al grupo de los de mayor tamaño y mejores resultados en la región. En Centro y Sudamérica, respectivamente, Costa Rica y Uruguay presentan dimensiones que los vuelven casos especialmente comparables. Un desarrollo económico y social relativamente temprano, la consolidación de una institucionalidad democrática estable, y extensiones territoriales y poblacionales semejantes. También las inversiones en $\mathrm{I}+\mathrm{D}$ como porcentaje del PBI son similares para Costa Rica y Uruguay, con valores de $0.43 \%$ y $0.41 \%$, respectivamente, en 2016 - (StatNano). Venezuela, en cambio, es un caso especial e interesante pues si bien tenía una menor inversión en I+D como porcentaje del PBI - 0.12\% en 2016 - (StatNano), su productividad científica en los años ochenta del siglo pasado era comparable a la de los países de la región de mayor desarrollo relativo como México, Brasil y Argentina (Mercado et al., 2020: 16). Por otro lado, son similares en términos del PBI per cápita (en dólares constantes de 2010). En 2020, el valor de ese indicador era de 9,619.7 para Costa Rica y 13,430.7 para Uruguay; el último valor disponible para Venezuela, en 2014, era de 14,026.4 (World Bank, s/f). Por lo tanto, hemos integrado a Venezuela a la comparación con los dos países antes citados.

Las técnicas empleadas para la recolección de datos fueron la revisión de literatura científica, de informes de agencias nacionales y documentos de política, además de la base StatNano, especializada en nanotecnología. Hemos identificado algunos factores cuya descripción y análisis permitieron una comparación de: a) diseño de políticas, y, b) actividades de I+D.

Para la primera parte hemos utilizado los ejes: a.1) instrumentos de política y financiamiento; a.2) gobernanza y nanoseguridad. 
Para la segunda, hemos empleado algunos indicadores ${ }^{1}$ de StatNano (presentados entre paréntesis), para los siguientes ejes: b.1) producción científica (publicaciones relacionadas con nanotecnología; prioridad nacional de la nanociencia); b.2) visibilidad de la producción científica (proporción de publicaciones de nanotecnología en Q1 y proporción del 10\% superior de artículos del Q1; promedio de citas por artículo de nanotecnología); b.3) colaboración internacional (proporción de colaboración internacional en nanociencia); b.4) desempeño industrial (patentes de nanotecnología concedidas, en las oficinas de patentes europea - EPO, o de Estados Unidos -USPTO).

\section{Panorama de CTI y nanotecnología en los tres países CTI en Costa Rica}

La CTI en Costa Rica se encuentra representada por el Ministerio de Ciencia, Tecnología y Telecomunicaciones (MICITT) y el Consejo Nacional para Investigaciones Científicas y Tecnológicas (CONICIT). La inversión total de fondos para la investigación en I+D es baja. Para 2020 fue de apenas 13.5 millones de dólares, siendo MICITT el segundo ministerio con el menor presupuesto para ese año. Este resultado se explica por una reducción de inversión en I+D por parte del sector académico, específicamente en la asignación de recursos para el desarrollo de proyectos de I+D. En 2018, el país invirtió el 2.67\% del PIB en actividades de ciencia y tecnología (CyT), con un incremento del $0.31 \%$ respecto al 2017, lo que representaba el 0.39\% del PIB en I+D (gráfica 1).

Gráfica 1. Inversión en actividades de CyT y en I+D en Costa Rica (2014-2018).

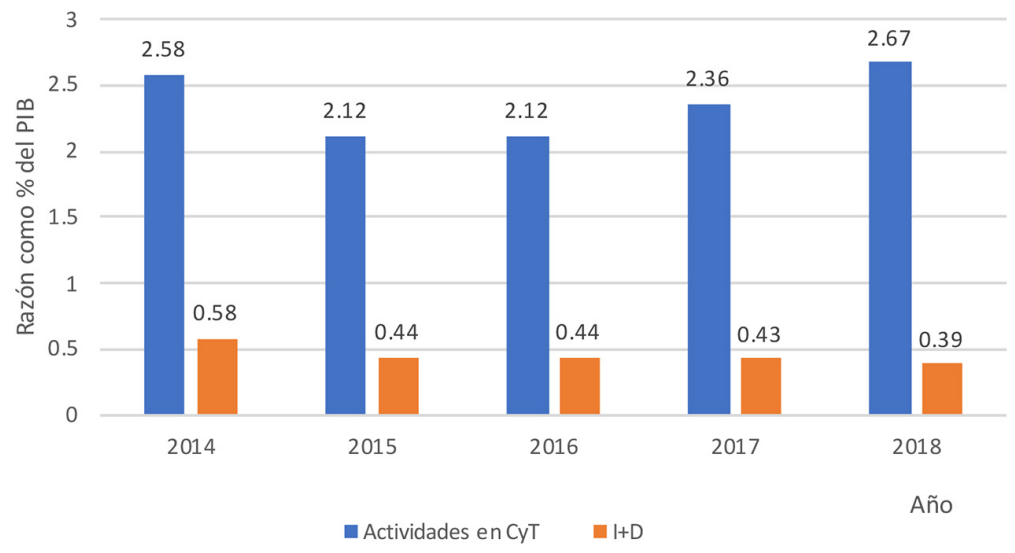

Fuente: MICITT/CONICIT, 2018.

\footnotetext{
${ }^{1}$ Cabe destacar que evaluar la I+D regional a lo largo del tiempo, a través de la medición de los artículos publicados en las revistas de mayor reconocimiento internacional, disponibles en plataformas de información científica como Web of Science y Scopus, en nanotecnología no presenta las dificultades de otros campos del conocimiento, cuyos investigadores eligen publicar en el medio local (RICYT, 2008).
} 


\section{Nanotecnología en Costa Rica}

Como respuesta al fenómeno mundial de la nanotecnología, en el año 1999 se concretó la creación del Centro Nacional de Alta Tecnología (CENAT), un órgano interuniversitario especializado en el desarrollo de investigaciones y posgrados en áreas de alta tecnología y de proyectos de vinculación e innovación tecnológica con el sector gubernamental y empresarial. CENAT fue creado al amparo del Convenio de Coordinación de la Educación Superior Universitaria Estatal, en la sesión del Consejo Nacional de Rectores (CONARE), número 5-99 de ese año. El objetivo primordial de CENAT es ejecutar actividades de capacitación, de investigación y servicios en ciencia y tecnología en varias áreas estratégicas y programas que permitan proveer al país de la tecnología pertinente para un desarrollo competitivo de los diferentes sectores de la sociedad en el ámbito económico, social y ambiental. Entre esas áreas estratégicas se encuentra el Área de Ciencia e Ingeniería de los Materiales y miniaturización de sensores, en la que con el apoyo de diversos sectores del país, MICITT, CONARE, CENAT y la industria de alta tecnología, así como de instituciones internacionales como la NASA, el 31 de agosto del año 2004, se inauguró el Laboratorio Nacional de Nanotecnología (LANOTEC) e inició labores de investigación el 18 de octubre del mismo año. LANOTEC fortaleció al país en investigación científica, brindándole la capacidad de ser el líder tecnológico en la región centroamericana y del Caribe, con ingeniería de punta en el estudio de materiales avanzados para la investigación, diseño y entrenamiento en tecnologías asociadas con la microtecnología, nanotecnología y ciencia de los materiales. Permitió además, ampliar el desarrollo de conocimiento y colaborar con formación de capital humano y la investigación científica en esta área. También contribuyó a desarrollar aplicaciones específicas para el sector productivo en diferentes tipos de industrias como la metalúrgi$\mathrm{ca}$, la de los materiales, la de los polímeros, para la microbiología, la medicina, la geofísica y la exploración espacial, entre otras. Estos conocimientos y futuras innovaciones en diversas aplicaciones tienen un gran potencial comercial y de desarrollo económico para el país y sus colaboradores. Considerando lo anterior, este laboratorio, como un ente que proyecta su liderazgo en la región, debería poseer un norte dirigido hacia seis nodos temáticos que incluyen la investigación, la extensión, la docencia, acciones en innovación y emprendedurismo, el deporte y el arte. En la tabla 1 se presentan algunas de las áreas de trabajo que se desarrollan en el nodo de investigación. Todo esto se complementa con una Unidad de Prestación de Servicios, que brinda apoyo técnico-científico a las empresas que así lo requieran.

LANOTEC cuenta con una inversión de más de 8 millones de dólares en equipamiento, incluyendo microscopios SEM y TEM, así como de AFM.

A continuación, se resumen el nacimiento y desarrollo de la nanotecnología en Costa Rica:

- 2004 - Creación de LANOTEC. 
- Se incluyen la bio y la nanotecnología como pilares de desarrollo nacional en la Estrategia del Siglo XXI.

- 2011- Decreto N. ${ }^{\circ}$ 36567-MICITT: "Declaratoria de interés público de la investigación en nanotecnología y sus aplicaciones".

- Existencia de programas y redes universitarias de CONARE: POLIUNA, CICIMA.

- Incorporación de LANOTEC a la Secretaría Técnica para la Gestión de Sustancias Químicas: Comisión de Nanotecnología: MS, MINAE, CONARE.

- Creación del Comité de Productos Químicos y Biotecnología de OCDE Costa Rica.

- 2013 - Creación del Programa Nacional de Desarrollo, PNDN.

- 2016 - Cofundación del Clúster Costarricense de Biotecnología, Dispositivos Médicos y Ciencias de la Vida, CRBiomed.

- 2016 - Incorporación de LANOTEC A ILSI Mesoamérica. En la actualidad tiene la presidencia.

Tabla1. Nodos de trabajo en LANOTEC-CENAT.

\begin{tabular}{|c|c|c|c|}
\hline Número & Nodo & Temas & Objetivo \\
\hline 1 & $\begin{array}{l}\text { LANOTEC } \\
\text { FABLAB }\end{array}$ & Procesos de innovación & Procesos de innovación \\
\hline 2 & $\begin{array}{l}\text { Biorrefinería y } \\
\text { economía circular }\end{array}$ & $\begin{array}{l}\text { Bioeconomía } \\
\text { Biorrefinería: biomasa, } \\
\text { energía. } \\
\text { Materiales, nanomateriales, } \\
\text { polímeros y plásticos, } \\
\text { composites. }\end{array}$ & Procesos de innovación \\
\hline 3 & Nanobiodiversidad & $\begin{array}{l}\text { Nanobiotecnología y } \\
\text { nanobiomimetismo }\end{array}$ & Procesos de innovación \\
\hline 4 & Ciencias de la vida & $\begin{array}{l}\text { Nanomedicina, } \\
\text { dispositivos médicos, } \\
\text { Farmacia } \\
\text { Proyectos de alta } \\
\text { tecnología: IoT, IoMT }\end{array}$ & Procesos de innovación \\
\hline 5 & $\begin{array}{l}\text { Vocaciones } \\
\text { científicas }\end{array}$ & $\begin{array}{l}\text { Nanoprofesor, OLCOQUIM, } \\
\text { OLCOCI }\end{array}$ & Procesos de innovación \\
\hline 6 & $\begin{array}{l}\text { Regulación e } \\
\text { impacto en la } \\
\text { sociedad }\end{array}$ & $\begin{array}{l}\text { Acreditación ISO } 17025 \\
\text { Nanometrología, patrones } \\
\text { de referencia. } \\
\text { Normalización, seguridad } \\
\text { laboral. } \\
\text { Aspectos regulatorios, } \\
\text { OCDE. }\end{array}$ & Procesos de innovación \\
\hline
\end{tabular}

Fuente: Elaboración propia, 2016. 
- 2019 - Creación del CTN 060 de Nanotecnología de INTECO.

- Participación en la creación de la Estrategia Nacional de Bioeconomía 2020-2030.

\section{CTI en Uruguay}

A partir del año 2007, Uruguay puede ser caracterizado por la definición de políticas públicas, planes e instrumentos para el desarrollo de la CTI. En un contexto de crecimiento económico, se crearon nuevas estrategias para el estímulo de la investigación y la innovación en el país con un cierto nivel de asignación de recursos por parte del Estado uruguayo, los que se suman al aporte, en menor proporción, del sector privado. La evolución de los valores de la inversión en I+D como porcentaje del PBI en el periodo 2009-2018 (tabla 2), indica que en Uruguay esta inversión no se ha mantenido constante. Este gasto está atrasado en comparación regional e internacional (RICYT, 2020). Por otro lado, la participación del sector privado no llegaba en el año 2015, a una cuarta parte de la inversión (ANII, 2017: 15).

En Uruguay las actividades de I+D se realizan mayoritariamente en el sector público, en la Universidad de la República (UDELAR), el Instituto Nacional de Investigaciones Agropecuarias (INIA) y el Instituto de Investigaciones Biológicas Clemente Estable (IIBCE).

Tabla 2. Inversión de Uruguay en I+D como porcentaje del PBI (2009-2018).

\begin{tabular}{|c|c|c|c|c|c|c|c|c|c|c|}
\hline Año & 2009 & 2010 & 2011 & 2012 & 2013 & 2014 & 2015 & 2016 & 2017 & 2018 \\
\hline $\begin{array}{c}\text { I+D/ } \\
\text { PBI (\%) }\end{array}$ & $0.41 \%$ & $0.34 \%$ & $0.35 \%$ & $0.33 \%$ & $0.32 \%$ & $0.34 \%$ & $0.36 \%$ & $0.41 \%$ & $0.49 \%$ & $0.42 \%$ \\
\hline
\end{tabular}

Fuente: Elaboración propia con base en RICYT, 2020.

Uruguay contaba en el año 2018 con 2,402 investigadores, 67.2\% de los cuales tenía un doctorado. A pesar de esto, para desarrollar un modelo competitivo, de diferenciación y diversificación, que agregue valor y conocimiento a la economía tradicional, es necesario un cambio de tendencia que conduzca a aumentar el número de investigadores, por lo menos al triple (Pohl Consulting y Asoc., 2016). Es débil la relación entre academia y sector productivo, así como también entre oferta de instrumentos y demandas de innovación basadas en ciencia y tecnología.

\section{Nanotecnología en Uruguay}

En el año 2009 el gobierno uruguayo, como ya lo habían hecho otros muchos países de la región, incluyó la nanotecnología como área transversal prioritaria en su programa estratégico de ciencia, tecnología e innovación (CTI). Previamente a esta definición de política pública, un conjunto de diecisiete investiga- 
dores locales había creado, en el 2006, el grupo G-Nanotec-Uy que integraba los distintos equipos que ya habían iniciado actividades de I+D en el país. Estos grupos pertenecían a UDELAR, a excepción de un equipo del IIBCE (Chiancone, 2012).

A partir de esa situación inicial, surgieron nuevos grupos y comenzó a desarrollarse I+D en otras instituciones. Entre ellas, el Instituto Pasteur de Montevideo (IPMO), inaugurado a fines de 2006; el Grupo de Tecnología de Proteínas del Laboratorio de Biotecnología de la Universidad ORT Uruguay, creado en el 2010, y, el Laboratorio Tecnológico del Uruguay (LATU).

Por otro lado, nuevas líneas de trabajo se agregaron a las iniciales, fundamentalmente en áreas como salud y energía. El proceso de diversificación ha estado asociado con la colaboración interinstitucional en proyectos, además a un fuerte relacionamiento internacional de los investigadores.

Un evento altamente reconocido y difundido ha sido el establecimiento de la "primera unidad de investigación especializada en nanotecnología del país". NanoMat, el laboratorio de nanotecnología del Polo Tecnológico de Pando (PTP) fue creado con el apoyo del programa Uruguay Innova, donación de la Unión Europea. Esos fondos y los recursos de un proyecto ANII permitieron la adquisición de equipo científico tecnológico. El Instituto PTP es desde el año 2012 una Unidad Académica de la Facultad de Química de UDELAR, dedicada a la investigación, desarrollo e innovación, en química, biotecnología, ciencias de los materiales y medio ambiente. Su especificidad radica en estar orientada desde su origen por la demanda del sector productivo de bienes y servicios, tanto privado como público (Polo Tecnológico, s/f).

La necesidad de formar recursos humanos altamente capacitados en nanotecnología ha estado presente desde las primeras etapas, en las consideraciones de los investigadores uruguayos. Esta inquietud se ha concretado en una propuesta de maestría en nanociencia y ciencia de materiales del Programa de Desarrollo de Ciencias Básicas (PEDECIBA), aprobada en 2018.

\section{CTI en Venezuela}

Desde inicios de este siglo, el sistema venezolano de CTI se ha visto seriamente afectado por las políticas en esa materia. A grandes rasgos estas políticas podrían caracterizarse como un intento del Estado por ejercer mayor control político sobre las universidades e institutos de investigación, lo cual ha generado fuertes tensiones políticas a partir del año 2002, resultando en la creación de una estructura paralela para la educación superior y lo que algunos autores han calificado como una "asfixia presupuestaria" a las universidades (Mercado et al., 2020: 24).

Esta situación empeoró considerablemente debido al fenómeno de hiperinflación que experimenta el país desde 2015, lo cual ha profundizado el colapso de la universidad pública venezolana. Como resultado, la inversión en CTI se ha visto drásticamente reducida. De acuerdo con datos oficiales del Observatorio Nacional de Ciencia y Tecnología e Innovación (ONCTI), del total 
del presupuesto anual que asigna el Ejecutivo a instituciones de educación superior, el porcentaje destinado a investigación no llega siquiera al 10\% en el mejor de los casos.

Otra consecuencia de la crisis es la pérdida de capacidades nacionales para CTI. La pérdida real de talento humano es difícil de estimar debido a la falta de cifras oficiales confiables, aunado a que muchos investigadores han solicitado jubilaciones anticipadas y permisos no remunerados, como una estrategia para intentar ubicarse en otros espacios con mejores retribuciones. Se estima que en los últimos 18 años emigraron 2,084 investigadores, la mayoría de los cuales lo hizo después de 2014 (Diez et al., 2020).

En Venezuela, la relación entre academia y sector productivo ha estado históricamente asociada con la prestación de servicios. Sin embargo, en los últimos años la capacidad de las instituciones de prestar estos servicios se ha visto disminuida, mientras que el sector productivo también registra una disminución de sus demandas de análisis y asistencia técnica hacia las universidades. En general, la situación actual del sistema nacional de CTI implica la pérdida de talento humano; obsolescencia de equipos, falta de materiales y suministros para la investigación; cierre de programas de posgrado y becas, deterioro de infraestructura, etcétera.

\section{Nanotecnología en Venezuela}

Venezuela no tiene una política pública específica para la nanotecnología, pero en las últimas dos décadas se han llevado adelante algunas iniciativas que favorecieron su desarrollo. En el año 2005, el Estado lanzó el Plan Nacional de Ciencia, Tecnología e Innovación (PNCTI 2005-2030), que reconoce explícitamente la importancia de la nanotecnología, pero no la incluye en la agenda de prioridades de investigación. En el año 2009, la Red Venezolana de Nanotecnología (RedVnano) presentó al entonces Ministerio del Poder Popular para Ciencia, Tecnología e Industrias Intermedias, una propuesta de Plan Nacional de Nanotecnología a 10 años, estructurado a partir de una alianza Red-Gobierno, con objetivos comunes para fortalecer el desarrollo endógeno y sustentable.

Cuatro instituciones son responsables de más del $80 \%$ de las publicaciones científicas en el campo nano: el Instituto Venezolano de Investigaciones Científicas (IVIC) (27\%), Universidad Central de Venezuela (UCV) (22\%), Universidad Simón Bolívar (USB) (21\%) y Universidad de Los Andes (ULA) (16\%) (López et al., 2015: 114). En cuanto a líneas de investigación, un análisis de las estructuras disciplinarias de las publicaciones de nanotecnología venezolanas entre 1975 y 2018 (figura 1), identificó que ciencias de materiales es el área predominante en todo el periodo, así como fisicoquímica, química analítica, fisicomatemáticas, bioquímica y biología molecular. En los últimos veinte años observamos nuevas áreas disciplinarias como las ingenierías, con una diversidad de aplicaciones en las áreas química, biomédica, materiales, entre otras (López et al., 2020). 
Figura 1. Estructura disciplinaria de las publicaciones en nanociencia y nanotecnología en Venezuela (1975-2018).

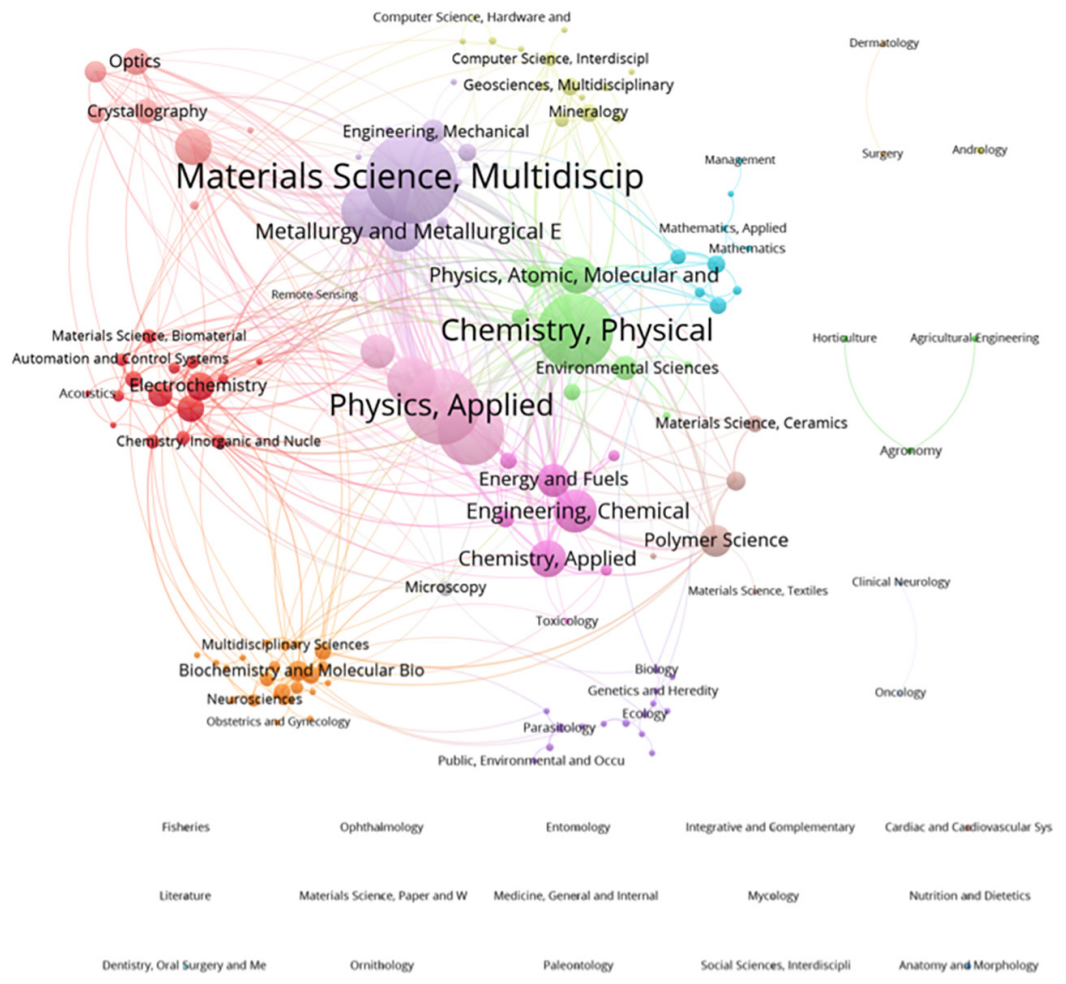

Fuente: López et al. 2020.

Según los datos obtenidos por la RedVnano para el año 2015, la mayoría de los equipos se encuentran en las principales instituciones académicas y algunos en la industria, concentrándose geográficamente en las ciudades de Caracas, Mérida, Maracaibo, Cumaná y Puerto Ordaz (López et al., 2015). La infraestructura disponible para nanocaracterización y nanofabricación incluye equipos para análisis químicos, tamaño de partícula y recubrimientos nanoestructurados, microscopios de sonda de barrido, SEM, TEM, difracción de rayos $\mathrm{X}, \mathrm{RMN}$, entre otros. Actualmente es difícil conocer las condiciones de operatividad de estos equipos, debido a la falta de presupuesto para su mantenimiento o incluso la disponibilidad de técnicos especializados en su manejo en las instituciones en las que se encuentran.

En cuanto a recursos humanos, los únicos datos disponibles son los recabados por la RedVnano. Para el momento de su creación la red agrupaba 106 miembros y ya para el año 2015 — fecha del último reporte- había alcanzado los 473 integrantes. Los cuales contaban con diferentes grados de formación académica y pertenecían a diversas instituciones principalmente 
públicas tanto del ámbito académico, como del ámbito productivo (Siderúrgica del Orinoco Alfredo Maneiro, Corporación Venezolana de Guayana y Petróleos de Venezuela); así como también del sector militar (Dirección de Tecnologías de Información y las comunicaciones del Ejército).

\section{Revisión comparativa Diseño de políticas}

Instrumentos de política y financiamiento

La mayoría de los fondos empleados para I+D en nanotecnología en Costa Rica provienen de los Fondos para la Educación Estatal Superior (FEES), provenientes de las universidades estatales. Un bajo porcentaje de proyectos de este campo corresponde a los aprobados por el MICITT y el CONICIT, así como a iniciativas privadas, especialmente en el sector de ciencias de la vida, alimentos y de materiales.

El comienzo de muchas de las líneas de investigación en nanotecnología en Uruguay fue sin financiamiento específico; investigadores guiados por su interés y consideraciones sobre los potenciales desarrollos. Sin embargo, la condición de área estratégica transversal atribuida posteriormente en el PENCTI brindó a las actividades de I+D, así como a la formación en nanotecnología, ciertas posibilidades de financiamiento. A estas se sumaron las diferentes convocatorias por fondos sectoriales (salud, energía) de la Agencia Nacional de Investigación e Innovación (ANII) y otras organizaciones (Chiancone, 2012). UDELAR, a través de las convocatorias de la Comisión de Investigación Científica (CSIC), representa otra fuente de recursos nacionales en los que participan los nanotecnólogos uruguayos.

Tampoco en Venezuela existe una política oficial en materia de nanotecnología. Sin embargo, el Estado ha financiado y acompañado varias iniciativas surgidas desde la RedVnano. Entre los convenios más importantes encontramos el Programa de Cooperación de Postgrados (PCP) y el programa PREFALC NANO2, que cofinanciaron la movilidad de estudiantes e investigadores entre Venezuela y países como Francia, Argentina y Brasil. El convenio Cuba-Venezuela en su apartado sobre ciencia y tecnología financió no solo actividades de formación sino también proyectos de investigación (López et al., 2015).

En el año 2014, se llevó a cabo el Foro NanoSur con un fuerte apoyo del Ministerio de Ciencia y Tecnología (Mincyt). El evento reunió investigadores, representantes oficiales y del sector productivo tanto de Venezuela, como de varios países de América Latina, Europa y nuevos aliados políticos como Irán. El objetivo del Foro era impulsar la creación de la Comunidad de Nanotecnología del MERCOSUR (NanoSur), una propuesta que presentó Venezuela en el marco de la Reunión Especializada en Ciencia y Tecnología del MERCOSUR (RECyT) del año 2013, para promover la cooperación regional en el área. Sin embargo, esta propuesta tampoco prosperó en el seno de la organización.

En cuanto al financiamiento, la mayor parte de las actividades de investigación se realizan con financiamiento público, a través del presupuesto or- 
dinario de las instituciones y de convocatorias generales de Mincyt. La Red NANOANDES genera cada año una escuela de nanotecnología a nivel latinoamericano, con fuerte participación de Francia, además de Costa Rica, Brasil, México, Argentina, Perú, Ecuador, Bolivia, entre otros países.

\section{Gobernanza y nanoseguridad}

En la actualidad, LANOTEC está siendo acreditado en las Normas ISO 170252017 con el fin de cumplir con todos los requerimientos que exigen las empresas del sector de ciencias de la vida. Asimismo, desde hace cuatro años se inició un proceso de normalización de la nanotecnología en Costa Rica, junto con el Instituto de Normas Técnicas de Costa Rica (INTECO). Otro objetivo para el 2021, es poder ser parte del Comité Técnico en Nanotecnología de la Organización Internacional de Estandarización, el ISO/TC-229, y continuar el proceso de establecer regulaciones de la nanotecnología en Costa Rica. Como parte de la incorporación de Costa Rica a la Organización para la Cooperación y el Desarrollo Económicos (OCDE), LANOTEC fue invitado a formar parte del Working Party on Manufactured Nanomaterials (WPMN), para colaborar con el Comité de Productos Químicos y Biotecnología de OCDE Costa Rica. También para promover la cooperación internacional en los aspectos de salud humana y seguridad ambiental de los nanomateriales manufacturados, y otros materiales avanzados para la reglamentación, considerando esquemas de gestión voluntarios, legislativos o de otro tipo. Se espera trabajar en los seis tipos más comunes de formas voluntarias de vigilancia de la nanotecnología, conocidas como "regulación blanda": registros, etiquetado, códigos de conducta, sistemas de manejo de riesgo, guías y estándares técnicos.

En Uruguay se ha desarrollado un conjunto de reuniones, cursos, talleres de difusión sobre nanotecnología y los aspectos éticos, legales y sociales (ELSA), así como de los potenciales riesgos para la salud y el ambiente. En 2012, el país participó voluntariamente junto a Nigeria y Tailandia, en un proyecto piloto del Instituto de las Naciones Unidas para la Formación Profesional y la Investigación (UNITAR) sobre la seguridad en la gestión de productos de nanotecnología. Entre los elementos identificados sobre la nanoseguridad en Uruguay, se encontraba la escasa visibilidad del tema; debilidades en el manejo de sustancias químicas; necesidad de incorporar los aspectos ELSA; y requerimientos de equipamiento y personal calificado (Chiancone y Martínez Larrechea, 2015).

La subcomisión de nanometrología y nanoseguridad del Consejo Sectorial de Nanotecnología (del Ministerio de Industria, Energía y Minería (MIEM)) estableció un conjunto de objetivos que incluyen:

- El estímulo a la capacitación de personal técnico y formación de nuevos doctorandos en el área.

- La organización de recursos materiales y humanos para implementar los protocolos en Uruguay. 
- La realización de ejercicios de ensayos de aptitud entre laboratorios nacionales, y la participación en estos ejercicios con países de la región.

- $\quad$ El fomento de regulaciones de productos que contengan material nanoparticulado (registro, etiquetado, advertencias, composición y contenido).

- La armonización de técnicas en el ámbito del MERCOSUR y con otros bloques comerciales (Méndez, 2015).

En ese contexto se desarrolla el trabajo de nanometrología del grupo del Laboratorio de Biomateriales, de la Facultad de Ciencias (UDELAR) y el Laboratorio Tecnológico del Uruguay (con rol, entre otros, de Instituto Metrológico Nacional). Este trabajo cuenta con el apoyo de instituciones como el MIEM, la CSIC (UDELAR), la ANII, y el PEDECIBA.

En el área de gobernanza, en Venezuela se han logrado tímidos avances con la realización de mesas de trabajos, reuniones y eventos con el sector petrolero, metalúrgico y agroalimentario. Además, se han realizado reuniones y talleres con representantes del Instituto Nacional de Higiene Rafael Rangel (INHRR) y el Servicio Autónomo Nacional de Normalización, Calidad, Metrología y Reglamentos Técnicos (Sencamer), organismos públicos encargados de la regulación y control de diversos productos, bienes y servicios (López et al., 2020).

Entre 2014 y 2015 se desarrolló un proyecto bilateral en el marco del Convenio Cuba-Venezuela para avanzar en reglamentos, metrología y nanotoxicología en el área de salud en ambos países, cuyo producto más importante fue el primer documento regulatorio sobre la comercialización de productos farmacéuticos con nanotecnología en Venezuela. Se trata de una circular emitida por INHRR, donde se establece que todas las especialidades farmacéuticas que contengan nanotecnología se consideran productos nuevos para efectos de su evaluación y registro sanitario nacional.

\section{Actividades de I+D \\ Producción científica}

\section{Publicaciones relacionadas con nanotecnología}

La gráfica 2 permite comparar la evolución del número de artículos relacionados con nanotecnología, indexados en la Web of Science entre 2012 y el primer semestre 2021. La evolución de las publicaciones de los tres países en la última década presenta desempeños muy diferentes. Mientras que en el año 2012 Venezuela lideraba con 55 artículos, frente a 31 de Uruguay y 8 de Costa Rica, en 2020 (y desde 2017) el liderazgo le corresponde a Uruguay, con 72 artículos, mientras que Venezuela registraba 51 y Costa Rica 36. Esa tendencia se mantiene en el primer semestre de 2021.

El aumento de la capacidad de publicar y el número de las publicaciones de Costa Rica está asociado con distintos factores. En primer lugar, LANOTEC (el 
Gráfica 2. Publicaciones relacionadas con nanotecnología (artículos).

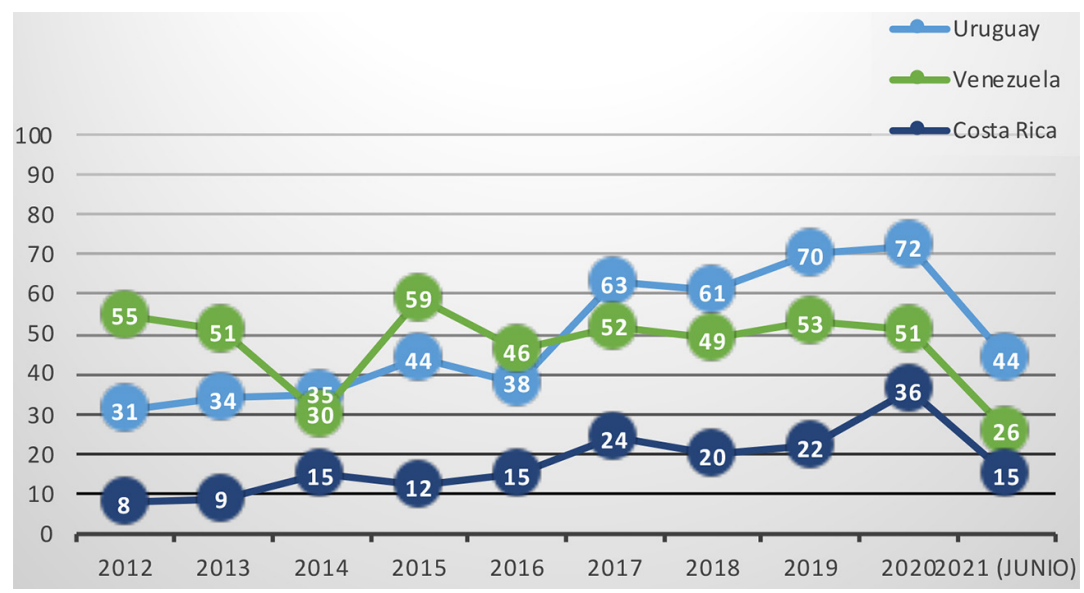

Fuente: Elaboración propia con base en StatNano (28/7/2021 a).

laboratorio de mayor capacidad en el país) aumentó durante ese periodo, la cantidad de equipos de alta tecnología para realizar caracterización. Entre esos equipos se encuentran los microscopios electrónicos de barrido (SEM) y de transmisión (TEM), lo cual redundó en una mayor calidad de las publicaciones e impulsó a LANOTEC a generar alianzas estratégicas y de colaboración.

Por otro lado, en LANOTEC también se dio un aumento de recursos humanos con un mayor grado académico. En el año 2021, el 90\% de los investigadores y colaboradores tienen el doctorado, con una formación básica en diversas áreas de las ciencias naturales y experimentales, así como en las ingenierías.

También en el caso de Uruguay, se ha dado un crecimiento del número de publicaciones indexadas, alcanzándose en el primer semestre de 2021, la misma cantidad de artículos que en todo el año 2015. Diversos factores apoyaron el desarrollo del campo de la nanotecnología en el país, que explicaría dicho aumento. Entre ellos, el proceso de consolidación y diversificación temática de los grupos de I+D en las distintas instituciones (UDELAR, PTP, ORT, IPMO y LATU), el acceso a fondos a través de las convocatorias locales y la cooperación internacional, con los consecuentes progresos en la formación de recursos humanos y la adquisición de equipamiento científico. Las colaboraciones científicas con investigadores de otros países aportan en la misma dirección.

En el caso de Venezuela, se observa que el ritmo de publicaciones es inestable en el periodo analizado, aunque se evidencia el mantenimiento de cierta capacidad para publicar, a pesar de los recortes en el financiamiento y los efectos negativos de la crisis que sufre el país desde el año 2015. Esto se puede explicar en parte por el alto grado de colaboración que caracteriza el campo, lo cual permite a los investigadores nacionales apoyarse en la infraestructura y demás recursos de sus colaboradores en el exterior. 
Otro factor que contribuye positivamente a esta capacidad es que muchos investigadores han desarrollado estrategias para ubicarse en institutos de investigación en el exterior y así obtener financiamiento mediante diversas figuras, sin abandonar formalmente su institución de adscripción en Venezuela. Unas de las figuras más comunes son las estancias de investigación, permisos no remunerados y años sabáticos.

\section{Prioridad nacional de la nanociencia}

Es un indicador que mide la prioridad nacional de la nanociencia y muestra la concentración de actividades científicas en esta área del conocimiento en un país determinado. Se define como la relación entre el cociente de artículos de nanotecnología de un país sobre el total global de artículos de nanotecnología, y el cociente de los artículos del país sobre el total global de artículos en el mismo periodo. La región Mundo es la unidad (StatNano, 28/7/2021 b).

La gráfica 3 permite observar que en la evolución de la prioridad nacional de la nanociencia de cada país (entre 2012 y marzo 2021), Uruguay comenzó con un valor de 0.55 en 2012 y terminó con 0.71 , con registros diferentes cada año. Venezuela, en cambio, pasó de 0.68 a 0.4 y Costa Rica partió de 0.17 en 2012 y alcanzó solo 0.08 en marzo de 2021. En resumen, la consideración de los valores del periodo muestra un cambio en las posiciones relativas de estos países, quedando al final Uruguay en primer lugar, en alza; Venezuela, en segundo lugar, a la baja y, por último, Costa Rica, parcialmente en alza, con picos en 2014, 2017 y 2020.

En marzo 2021, los valores del indicador para México, Brasil y Argentina eran 0.92, 0.73 y 0.64, respectivamente (StatNano 28/7/2021 b).

Gráfica 3. Prioridad nacional de la nanociencia.

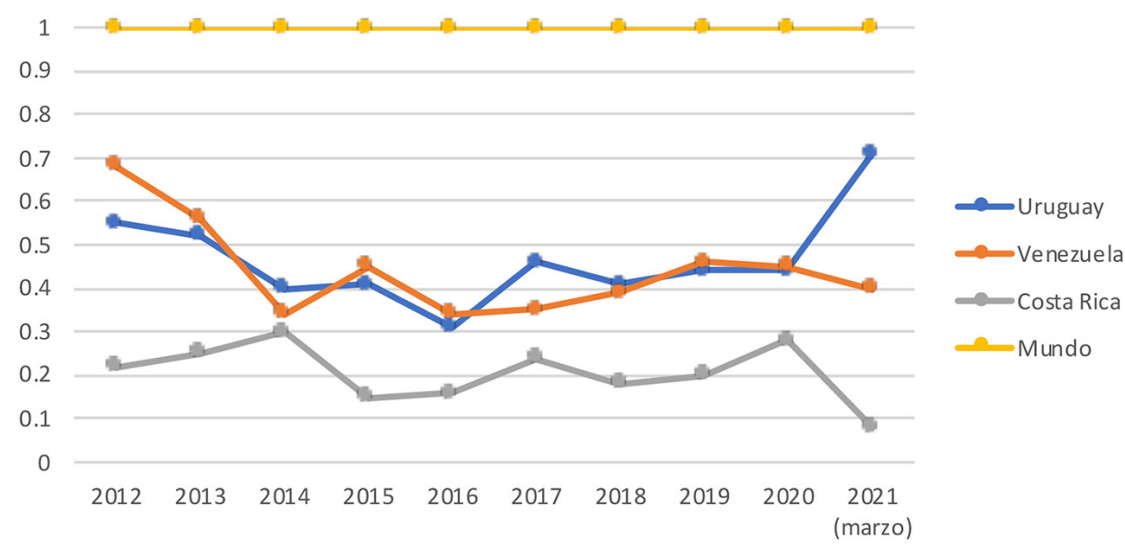

Fuente: Elaboración propia con base en StatNano (28/7/2021 b). 


\section{Visibilidad de la producción científica}

\section{Proporción de publicaciones de nanotecnología en Q1 y proporción} del $10 \%$ superior de artículos del Q1

En la tabla 3 se presentan los valores de dos indicadores. Por un lado, la participación en las publicaciones de nanotecnología del Q1. Este indicador expresa como porcentaje para cada país, la proporción de artículos de nanotecnología publicados en las revistas del primer cuartil (Q1), en relación con el total de artículos de nanotecnología publicados (StatNano 28/7/2021 c).

Por otro lado, la proporción de artículos de nanotecnología publicados en el $10 \%$ de las revistas superiores, en relación con el total de artículos de nanotecnología publicados en revistas del primer cuartil (Q1) (basado en la magnitud del factor de impacto - JCR) - (StatNano 28/7/2021 d).

Tabla 3. Participación en revistas del Q1 (\%) y en artículos del $10 \%$ superior del Q1, por año y por país (\%).

\begin{tabular}{|c|c|c|c|c|c|c|c|}
\hline Pais & 2014 & 2015 & 2016 & 2017 & 2018 & 2019 & 2020 \\
\hline Costa Rica - Q1 & 40 & 58.33 & 33.33 & 16.67 & 35.00 & 40.91 & 41.67 \\
\hline Uruguay - Q1 & 54.29 & 29.55 & 39.47 & 42.86 & 36.07 & 42.86 & 33.33 \\
\hline Venezuela - Q1 & 56.67 & 42.37 & 32.61 & 25.00 & 28.57 & 33.96 & 27.45 \\
\hline Mundo - Q1 & & & & & & & 48.39 \\
\hline Costa Rica - 10\% sup. Q1 & 16.67 & 42.86 & 20.00 & 25.00 & 28.57 & 33.33 & 26.67 \\
\hline Uruguay - 10\% sup. Q1 & 23.32 & 15.38 & 13.33 & 18.52 & 18.18 & 20.00 & 25.00 \\
\hline Venezuela - 10\% sup. Q1 & 35.29 & 16.00 & 20.00 & 15.38 & 14.29 & 16.67 & 7.14 \\
\hline Mundo - 10\% sup. Q1 & & & & & & & 34.74 \\
\hline
\end{tabular}

Fuente: Elaboración propia con base en StatNano (28/7/2021 c; 28/7/2021 d).

En cuanto al impacto y visibilidad de la producción de los tres países, revisando el primer cuartil, y en especial el $10 \%$ superior del primer cuartil, el liderazgo corresponde a Costa Rica. Su producción se destacó con el 58.33\% en el Q1 en 2015, con una baja en 2017 (16.67\%) y con 41.67\% en 2020.

A pesar de que la mayoría de las publicaciones de Costa Rica no se encuentran en el 10\% superior (tabla 3) muchas de estas están calificadas en el primer cuartil (Q1) de SCIMAGO, y este valor se ha ido incrementando conforme pasan los años, alcanzando un valor de 26.67 para 2020. Para el 2020, LANOTEC publicó 9 artículos en el primer cuartil (Q1), 11 artículos en el segundo cuartil (Q2), dos en el tercero (Q3) y 1 en el cuarto cuartil (Q4), y hasta abril del 2021, ya se han publicado 11 artículos, 8 de ellos en los dos primeros cuartiles (Q1 y Q2). Se espera que en 2021 este valor aumente, pues se han realizado publicaciones en el $10 \%$ de las revistas superiores de nanotecnología. 
Por otro lado, Uruguay y Venezuela participaban en Q1 con un $54.29 \%$ y $56.67 \%$ en 2014, pero en 2020 estaban claramente por detrás de Costa Rica (41.67\%), con $33.33 \%$ y $27.45 \%$, respectivamente.

El valor de la participación en Q1 de los tres países es menor que el promedio mundial del año 2020 (48.39\%). Sin embargo, las posiciones relativas de Costa Rica y Uruguay son superiores a la de México (con 32.22\%); la de Costa Rica también está por encima de la posición de Brasil, con 38.14\% (StatNano 28/7/2021c).

En relación con la participación en el $10 \%$ de revistas del primer cuartil, en 2014 Costa Rica presentaba el menor porcentaje (16.67\%) y Venezuela, el mayor (35.29\%), con una situación intermedia de Uruguay (26.32\%). En 2020, en cambio, Costa Rica posee un $26.67 \%$ en ese rango y Venezuela ha caído al $7.14 \%$, mientras Uruguay sube al $25 \%$.

Cabe señalar que para 2020, los valores de este indicador para Costa Rica y Uruguay superan los de Argentina (22.46\%), Brasil (21.85\%) y México (21.39\%), y todos se encuentran por debajo del promedio mundial anual (34.74\%) (StatNano 28/7/2021 d).

Promedio de citas de los artículos de nanotecnología

Este indicador se define como el promedio del número de veces que los artículos de nanotecnología publicados en un año han sido citados en el Journal Citation Reports (JCR) (StatNano 28/7/2021e).

En la gráfica 4 se presentan los valores para el periodo 2012-2019 para los tres países.

Al comienzo del periodo, el promedio de citas por artículo de Venezuela casi llegaba a 30 y Uruguay estaba rondando las 24.5 citas, mientras que Costa

Gráfica 4. Promedio de citas por artículo (citas por artículo).

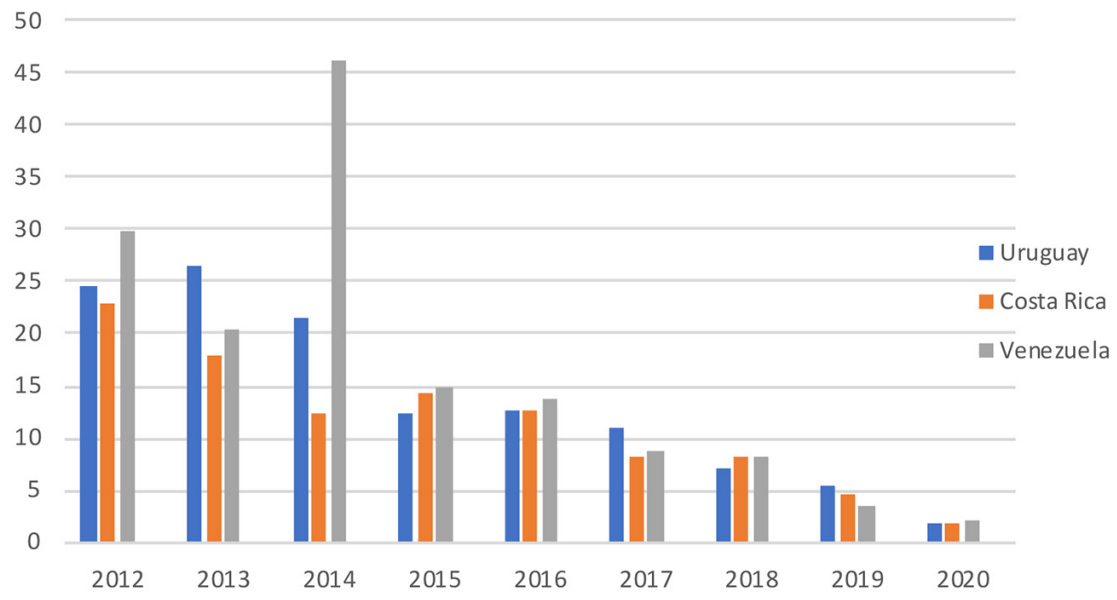

Fuente: Elaboración propia con base en StatNano (28/7/2021 e). 
Rica registraba algo menos de 23. A partir de 2015 en los tres casos, el desempeño de las citas decae marcadamente, con menos de 6 citas en 2019 y menos de 3 en 2020.

Los mayores países de la región también experimentaron el descenso antes mencionado. Los promedios de citas por artículo eran en 2020, algo superiores a los de Costa Rica, Uruguay y Venezuela: Brasil 2.59, México 2.06 y Argentina 3.01 (StatNano s/f e).

\section{Colaboración internacional}

\section{Proporción de colaboración internacional en nanociencia (\%)}

Este indicador mide el nivel de colaboración internacional entre los científicos de un determinado país, que tienen una actividad de publicación en nanociencia. Se define como el número de artículos en nanotecnología con autores de dos o más países, dividido entre el total de artículos del país en ese campo (StatNano 28/7/2021 f). El porcentaje de artículos de nanotecnología realizados en colaboración en el intervalo (2012-2020) se muestra en la gráfica 5.

Gráfica 5. Participación en colaboraciones internacionales (\%).

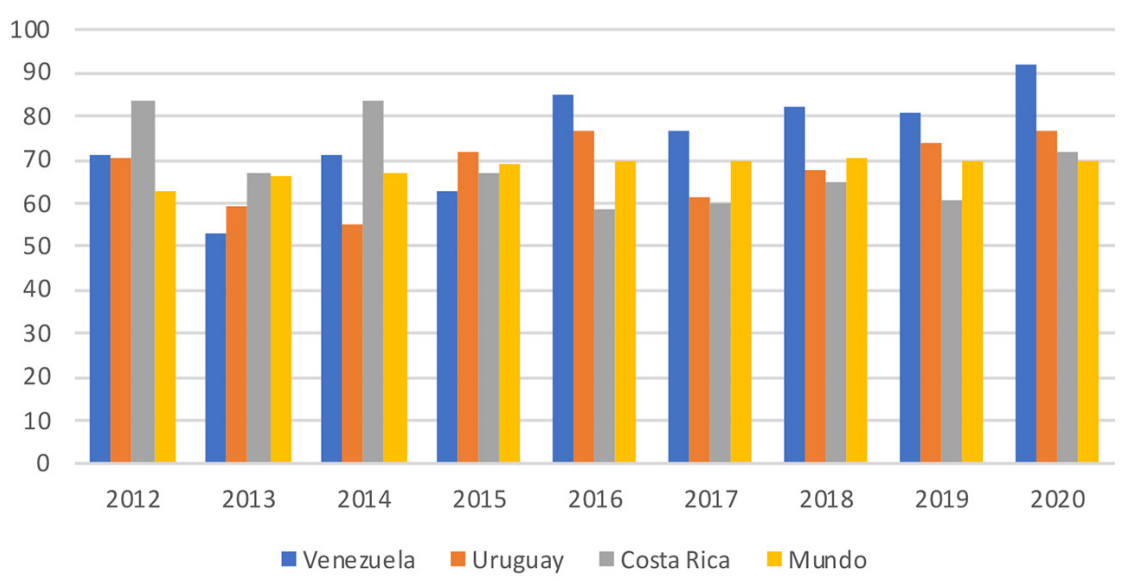

Fuente: Elaboración propia con base en StatNano (28/7/2021 f).

La participación de los tres países en las colaboraciones internacionales se mantuvo estable, con valores por encima del $50 \%$, cercanos y a veces superiores a los porcentajes mundiales. A partir de 2016, Venezuela tuvo la mayor participación colaborativa, alcanzando en 2020 un valor superior al 90\%.

Costa Rica que comenzaba el periodo superando al conjunto, se encontraba al final con el valor más bajo (algo mayor al 70\%). En cambio, Uruguay, que apenas superaba al inicio el $70 \%$, terminaba con una posición intermedia cercana al 76\%. 
Un relevamiento sobre colaboraciones internacionales en nanotecnología de varios países del MERCOSUR (Kay y Shapira, 2009) presentaba para Uruguay una distribución de la participación en publicaciones con coautorías de otros países, cuyos primeros cuatro valores correspondían a: i) colaboraciones de investigación con países latinoamericanos $-24.1 \%$ con Chile y $14.8 \%$ con Brasil, y, ii) colaboraciones con investigadores de países extrarregionales - 11.1\% con España; 11.1\% con Estados Unidos; y 9.3\% con Francia.

Otro estudio sobre redes de colaboración internacional hasta el año 2018 identificó los 10 países con los que Venezuela tiene mayor colaboración en publicaciones científicas nano, ubicando a Estados Unidos en el primer lugar con el $15.57 \%$, seguido de España y Francia (12.32\% y $10.81 \%$, respectivamente). También se encuentran entre los colaboradores, investigadores latinoamericanos y europeos (México, seguido de Inglaterra, Alemania, Brasil, Canadá, Argentina e Italia) (López et al., 2020).

La colaboración con grupos de otros países es parte de la dinámica científica actual y atraviesa todas las disciplinas. A su vez, los países pequeños entienden las colaboraciones internacionales como la mejor manera de robustecer su estructura de investigación científica (Belli y Balta, 2019: 39-40). Como en otros casos regionales en nanotecnología, esta estrategia se inscribe en los esfuerzos por superar las limitaciones locales, a través de la participación en redes de investigación internacionales y hacer convenios con laboratorios de otros países, alcanzando la masa crítica para lograr un buen desempeño en las actividades de I+D (Foladori, 2006; RICYT, 2008).

En esa dirección, cabe destacar que, los valores de este indicador en el mismo periodo para Brasil, Argentina y México son notoriamente menores que los tres casos analizados. En 2020, los porcentajes eran los siguientes: Brasil, 37.88\%, Argentina 53.55\% y México $44.87 \%$. El promedio mundial de ese año fue de 69.45\% (StatNano 28/7/2021 f).

\section{Desempeño industrial: patentes}

La norma ISO / TS 18110 (1a edición 2015-08-15), define las patentes de nanotecnología como aquellas que incluyen por lo menos una reivindicación relacionada con la nanotecnología que se pretende proteger, o que tiene uno de los códigos de nanotecnología de la clasificación IPC (StatNano 26/7/2021 g).

\section{Patentes de nanotecnología en EPO y en USPTO}

La gráfica 6 presenta el número de patentes de nanotecnología otorgadas por EPO y por USPTO en Costa Rica, Uruguay y Venezuela entre 2012 y junio de 2021.

De los tres países abordados, solamente Costa Rica se ha adherido al Tratado de Cooperación en materia de Patentes (PCT).

Las patentes de Costa Rica están relacionadas con un par de dispositivos médicos, un podómetro para medir pasos para personas de la tercera edad con 
Gráfica 6. Patentes de nanotecnología en EPO y en USPTO (patente).

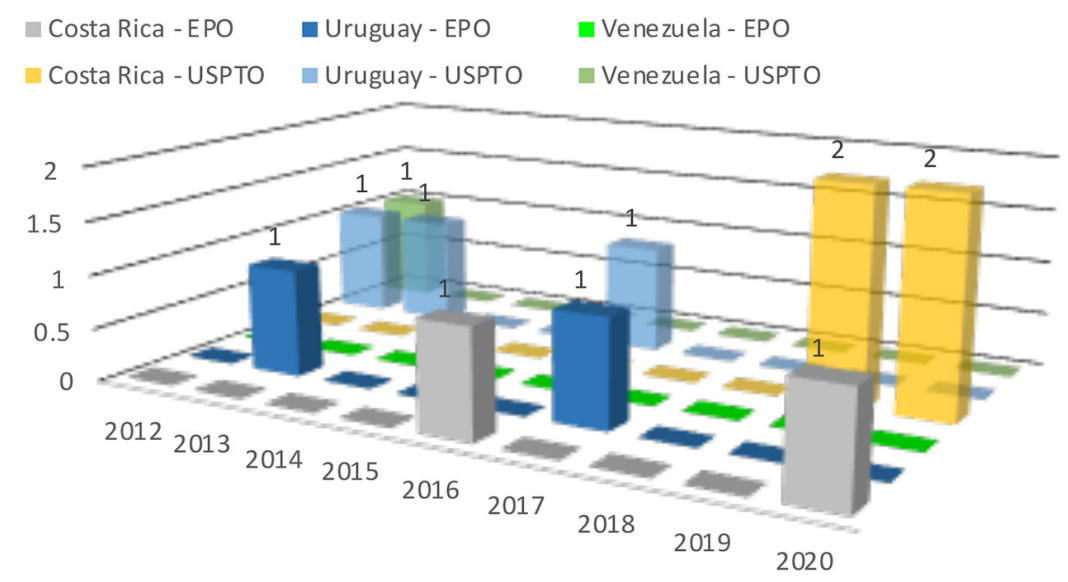

Fuente: Elaboración propia con base en StatNano (28/7/2021 g; 28/7/2021 h).

el fin de relacionar los resultados obtenidos con enfermedades crónicas. Este dispositivo posee tres sensores que permiten mejorar los valores obtenidos respecto a otros productos en el mercado. Está patentado en Estados Unidos, Costa Rica, Europa y Brasil. La segunda patente está vinculada con la obtención de nanoliposomas de quitosano empleados como mecanismos contra infecciones intrahospitalarias.

Uruguay es un país no adherido al PCT, y son varias las opciones para patentar que eligen los nanotecnólogos locales. Investigadores del Polo Tecnológico de Pando (PTP) han logrado patentar un proceso de preparación de materiales grafíticos magnéticos, desarrollado conjuntamente por científicos de la Universidad Federal de San Carlos, Brasil y UDELAR. Después de un periodo de algunos años en los que se solicitó patente bajo PCT, también ante EPO y la USPTO, en noviembre de 2012 la patente fue concedida por esta última oficina.

Además, el grupo del PTP solicitó en México un registro de patente PCT, de un material filtrante y filtro para retener hidrocarburos poliaromáticos, carbonilos y otros compuestos del humo de productos del tabaco y procesos industriales. Las instituciones financiadoras son UDELAR y una empresa local de tabaco. La patente fue solicitada en México por una compañía mexicana subsidiaria de la empresa uruguaya, por la universidad y por la tabacalera de Uruguay.

El caso de Venezuela, con una sola patente otorgada en los últimos 10 años, evidencia no solo la escasa tradición en el país de estas prácticas, sino también los efectos de la crisis en el área de desarrollo tecnológico e innovación. Las patentes en todas las áreas de conocimiento representan uno de los elementos más afectados, no solo por la crisis, sino principalmente por la po- 
lítica de protección de la propiedad intelectual aplicada por el Estado en los últimos quince años. Según un estudio realizado por De la Vega et al. (2008), basado en datos del Servicio Autónomo de Propiedad Intelectual (SAPI) de Venezuela, las primeras patentes en nanotecnología se registran en el año 1991 y ya para 2006 se registran 44 patentes otorgadas. Del total de solicitudes presentadas solo el $11 \%$ correspondía a instituciones venezolanas. Justamente en el año 2006 se produjo la salida de Venezuela de la Comunidad Andina de Naciones (CAN) que establecía un régimen de protección intelectual entre los países miembro. Esta decisión del gobierno venezolano significó un retroceso en esta materia para Venezuela y a partir de entonces, el SAPI implementó una política de abstención en el otorgamiento de patentes que se mantiene hasta hoy, bajo el argumento de que las patentes son instrumentos que limitan el conocimiento libre.

Estos casos están en consonancia con la situación de la región, donde son relativamente pocas las patentes de invención en la mayoría de los países (RICYT, 2008). En 2020, los valores de patentes concedidas en la EPO eran los siguientes: para Brasil 6, Argentina 0, y para México 2. En USPTO en cambio, la distribución era de 10 patentes para Brasil, 8 para México y 1 para Argentina StatNano (28/7/2021 g; 28/7/2021 h).

\section{Discusión final}

Durante las dos últimas décadas, el desarrollo de actividades de I+D en nanotecnología experimentó un proceso de expansión en los países abordados en este trabajo, en términos de la institucionalización del campo, la diversificación de líneas y grupos de investigación, y la producción científica y tecnológica.

En el diseño de las políticas públicas de estos países es posible reconocer, además de elementos comunes en las agendas de CTI y en los instrumentos de política, algunas variaciones nacionales.

Con base en las propias dinámicas disciplinarias, así como en decisiones asumidas desde fines de los años 90 del siglo pasado, se fue generando un ecosistema institucional de importancia, dando cuenta de la relevancia del campo en las agendas nacionales de CTI.

Diversas organizaciones (institutos, centros, laboratorios, redes, unidades académicas) han asumido la I+D en nanotecnología, con distintas líneas de investigación, con un creciente número de investigadores y con iniciativas de vinculación tecnológica con los sectores gubernamental y privado.

En el caso de Costa Rica se constata una creciente articulación interuniversitaria e interinstitucional. En Uruguay predomina una pauta fundamentalmente académica de organización de la nanotecnología (con la presencia destacada de la Universidad de la República), a la que se integran iniciativas de vinculación con el sector productivo en pos de desarrollos tecnológicos y patentes. En Venezuela, se ha generado el desarrollo paralelo de un nuevo sector gubernamental de educación superior, con el decaimiento y la asfixia 
financiera de las universidades públicas con mayor capacidad de investigación y con una fuerte emigración de científicos. Esto se expresó en una sensible caída del desempeño del país en el campo.

No existe en ninguno de los tres países una política específica de nanotecnología, más allá de su reconocimiento como área estratégica prioritaria en los programas nacionales de CTI.

Tampoco hay financiamiento destinado a la I+D en nanotecnología, salvo en Venezuela, donde la RedVnano logró el apoyo económico del gobierno para varias iniciativas de movilidad, formación avanzada e investigación en el sector.

El acceso a financiamiento y a instrumental de trabajo son cruciales en general en la investigación y muy particularmente en este campo. Sin un consistente apoyo estatal, las actividades de I+D podrían orientarse casi exclusivamente a las demandas del mercado, postergando una mayor conexión con demandas sociales, tanto o más estratégicas.

El conocimiento sobre los nanomateriales ha avanzado considerablemente, sin embargo, no tanto la evaluación de los potenciales riesgos contra la salud humana y el ambiente, así como su gestión. La falta de visibilidad detectada en Uruguay sobre el tema de la nanoseguridad, coincide con lo planteado tiempo atrás por Foladori e Invernizzi (2008) relativo a los escasos niveles de información pública y de participación, y a una práctica científica que se encapsula en un círculo reducido de actores.

En Costa Rica, se desarrolla desde hace cuatro años el proceso de normalización de la nanotecnología, incluido LANOTEC que está atravesando el proceso de certificación ISO 17025-2017. Se está participando en el desarrollo de mecanismos de soft-law (reglamentación blanda). En Uruguay, la iniciativa de UNITAR fue un estímulo a la discusión y consideración del tema por los nanotecnólogos y autoridades locales. En Venezuela no existen iniciativas de reglamentación de las nanotecnologías.

En cuanto a la producción científica considerada en términos de artículos indexados en la Web of Science, la evolución de los resultados de los tres países en la última década presenta resultados muy diferentes. Uruguay se posicionó a la cabeza de la producción, Costa Rica incrementó la misma y Venezuela decayó.

En relación con la prioridad nacional de la nanociencia, la evolución de los tres países en la década mostró un desempeño semejante. Uruguay mejoró en este indicador, Venezuela desmejoró y Costa Rica estuvo parcialmente en alza, con picos en determinados años.

En visibilidad internacional de la producción de los países estudiados, medida a partir de su presencia en revistas del primer cuartil, el liderazgo correspondió a Costa Rica en 2020, mientras que Uruguay y Venezuela estaban claramente por detrás. También en las revistas del $10 \%$ superior Costa Rica tiene una posición destacada a finales del periodo, por encima de los otros dos países. 
La producción de patentes de nanotecnología de los tres países en las oficinas internacionales (EPO y USPTO) también siguió pautas diferentes. El liderazgo relativo fue de Costa Rica, seguido por Uruguay y marginal el desempeño de Venezuela. El registro se verificó en la mayor parte de los casos en la oficina norteamericana.

Adicionalmente, nuestro estudio destaca que la participación de Costa Rica, Uruguay y Venezuela en las colaboraciones internacionales se mantuvo relativamente estable, con los mayores valores de participación colaborativa en Venezuela a partir de 2016. Los valores para estos tres países al final del periodo fueron superiores al porcentaje del mundo. Superaron también los valores de los mayores países de la región, Brasil, Argentina y México.

Dados los altos niveles de colaboración internacional de estos tres casos, consideramos la importancia de estudios futuros sobre su participación en redes con investigadores de diferentes países y las configuraciones de esas colaboraciones para los países con una menor concentración de actores y recursos en $\mathrm{I}+\mathrm{D}$.

Esta primera aproximación comparativa a la situación de la nanotecnología en Costa Rica, Uruguay y Venezuela, muestra que las últimas dos décadas han sido el marco temporal de un proceso de creciente institucionalización e internacionalización de la nanotecnología en los países considerados, en los cuales el desarrollo de este campo ha seguido fundamentalmente pautas nacionales, pero enfrenta desafíos comunes.

\section{Referencias}

ANII. (2017). Indicadores de ciencia, tecnología e innovación en Uruguay. Unidad de Evaluación y Monitoreo 2017. https://www.anii.org.uy/institucional/documentosde-interes/21/boletin-de-indicadores/

Chiancone, A. (2012). Nanociencias y nanotecnologías en Uruguay: áreas estratégicas y temáticas grupales. En Guillermo Foladori, Noela Invernizzi, y Edgar Záyago (eds.), Perspectivas sobre el desarrollo de las nanotecnologías en América Latina. México: M.A. Porrúa, 201-210.

Chiancone, A. y Martínez Larrechea, E. (2015). Caracterización del desarrollo de las nanotecnologías en Uruguay y el Plan Piloto de UNITAR. En: Guillermo Foladori; Anwar Hasmy, Noela Invernizzi y Edgar Záyago Lau (eds.), Nanotecnologías en América Latina: trabajo y regulación, 95-105. Ciudad de México: Miguel Ángel Porrúa.

Belli, S, y Balta, J. (2019). Mapeo de las publicaciones científicas entre América Latina, el Caribe y la Unión Europea. América Latina Hoy, 82: 7-41.

Cueto, M. (1989). Excelencia científica en la periferia. Actividades científicas e investigación biomédica en el Perú, 1890-1950. Lima: GRADE/ CONCYTEC.

De la Vega, I., Suárez, M., Blanco, F., Troconis, A. y Aponte, G. (2008). Las tecnologías nanoscópicas en los centros y las periferias. El caso de los nanomateriales en Venezuela. En Foladori, G. e Invernizzi, N. (coords.), Las nanotecnologías en 
América Latina. México: Miguel Ángel Porrúa, 125-146.

Diez, E., Freites, Y., García-Pérez, M., Ordóñez, L., Pineda, J., Requena, J. y Romero, S. (2020). Venezuelan research community migration: impacts and public policy implications. Banco Interamericano de Desarrollo. https://publications.iadb.org/ en/venezuelan-research-community-migration-impacts-and-public-policyimplications

Foladori, G. e Invernizzi, N. (coords.). (2008). Las nanotecnologías en América Latina. México: Miguel Ángel Porrúa.

Foladori, G., Invernizzi, N. y Záyago Lay, E. (orgs.). (2012). Perspectivas sobre el desarrollo de las nanotecnologías en América Latina. México: Miguel Ángel Porrúa.

Foladori, G. e Invernizzi, N. (2013). Inequality gaps in nanotechnology development in Latin America. Journal of Arts and Humanities, 2 (3): 35-45.

Foladori, G., Invernizzi, N. y Záyago Lau, E. (2016) Investigación y mercado de nanotecnologías en América Latina. México: Miguel Ángel Porrúa.

Hannerz, U. (2015). Center-periphery relationships. En James D. Wright (ed.), International Encyclopedia of the Social \& Behavioral Sciences. Elsevier, 308-311.

Invernizzi, Noela, Hubert, Matthieu y Vinck, Dominique. (2014). Nanoscience and nanotechnology: how an emerging area on the scientific agenda of the core countries has been adopted and transformed in Latin America. En Eden Medina, Ivan da Costa Marques y Christina Holmes (eds.), Beyond imported magic. Essays on science, technology, and society in Latin America. Cambridge, Massachusetts, Londres: The MIT Press, 223-242.

Kay, L. y Shapira P. (2009). Developing nanotechnology in Latin America. Journal of Nanoparticle Research, 11(2): 259-278. https://doi.org/10.1007/s11051-008-9503-z

Kreimer, P. (2010). Ciencia y periferia. Nacimiento, muerte y resurrección de la biología molecular en la Argentina. Buenos Aires: Eudeba.

López S. Trocel C., Hasmy A. y Vessuri, H. (2015). Estado actual de la nanociencia y nanotecnología en Venezuela. En Guillermo Foladori; Anwar Hasmy, Noela Invernizzi y Edgar Záyago Lau (eds.) Nanotecnologías en América Latina: trabajo y regulación. México: Miguel Ángel Porrúa, 111-134.

López, S. Robles-Belmont, E. y Pacheco, L. (2020). La investigación en nanoescala en Venezuela. A diez años de la propuesta del Plan Nacional de Nanotecnología. En Edgar Záyago Lau Guillermo Foladori y Noela Invernizzi (coords.), Relevancia social de las nanotecnologías en América Latina. México: Miguel Ángel Porrúa,157-177.

MICITT. (2018). X Informe de Indicadores Nacionales de Ciencia, Tecnología e Innovación. Costa Rica, San José: MICITT.

Matharan, G. (2017). La dinámica centro-periferia en el estudio de la ciencia en América Latina: notas para una reflexión historiográfica sobre la Argentina. El Hilo de la Fábula, (16): 33-48. https://doi.org/10.14409/hf.v0i16.6279

Méndez, E. (2015). Regulación de nanomateriales: visión de Uruguay. Presentación en el Taller Técnico sobre Nanotecnología y Nanomateriales, enfoque en Nanoseguridad América Latina y Caribe. Bogotá, Colombia, 22-24 June 2015. https:// www.unitar.org/media/2495 
Mercado, A., Ávalo, I., Sánchez-Rose, I., Cervilla, M., Autora y Vessuri, H. (2020). Doing research in Venezuela. Science, technology and innovation capabilities to overcome the crisis in Venezuela. Canadá, International Development Research Center (IDRC) y Global Development Network (GDN). http://www.gdn.int/ sites/default/files/u115/Venezuela\%20Report\%20Spanish\%20with\%20 ISBN.pdf

ONCTI. (2016). Indicadores venezolanos de ciencia, tecnología e innovación. Boletín año 2016. Observatorio Nacional de Ciencia, Tecnología e Innovación. Caracas, Ediciones ONCTI.

Pohl Consulting \& Asociados. (2016). Áreas de vacancia en I+D según la perspectiva del Sector Productivo. http://www.anii.org.uy/institucional/documentos-de-interes/25/ consultoria-fortalecimiento-del-sistema-nacional-de-innovacion

Polo Tecnológico. (s/f). ¿Qué es el Polo Tecnológico de Pando? http://www.polotecnologico.fq.edu.uy/es/que-es-el-polo-tecnologico-de-pando

RICYT. (2008). La nanotecnología en Iberoamérica. Situación actual y tendencias. http:// www.ricyt.org/wp-content/uploads/2019/09/Estado_2008_03Nano.pdf

RICYT. (2020). Uruguay 2007-2016. Recursos financieros I+D. http://dev.ricyt.org/ui/ v3/bycountry.html?country=UY\&subfamily=CTI_IMD

StatNano. (28/7/2021a). Nanotechnology publications (article). https://statnano. com/report/s29

StatNano. (28/7/2021b). National priority in nanoscience. https://statnano.com/report/s41

StatNano. (28/7/2021c). Share of Q1 nanotechnology publications (percent). https:// statnano.com/report/s143

StatNano. (28/7/2021d). Share of top 10\% nano-articles of Q1 nano-articles (percent). https://statnano.com/report/s145

StatNano. (28/7/2021e). Average citation per nanotechnology article (citations). https:// statnano.com/report/s55

StatNano. (28/7/2021f). Share of international collaboration in nanoscience (percent). https://statnano.com/report/s82

StatNano. (28/7/2021g). Nanotechnology patents in EPO (patent). https://statnano. com/report/s102

StatNano. (28/7/2021h). Nanotechnology patents in USTPO (patent). https://statnano. com/report/s103

Vega Baudrit, J. (2016). Plan Estratégico de Desarrollo 2016-2020. San José: LANOTEC. World Bank. (s/f). GDP per capita (current US\$). https://data.worldbank.org/indicator/ NY.GDP.PCAP.KD 